\title{
Best of lung cancer session presented during ERS 2017 Chinese language day: lung cancer screening
}

\author{
Clementine Bostantzoglou, Chrysoula Kontogianni, Marianthi Iliopoulou, Mina Gaga \\ 7th Respiratory Medicine Department, Sotiria Athens Chest Hospital, Athens, Greece \\ Correspondence to: Mina Gaga. 7th Respiratory Medicine Department, Sotiria Athens Chest Hospital, 152 Mesogion Avenue, Athens 11527 , Greece. \\ Email: minagaga@yahoo.com.
}

Submitted Sep 25, 2017. Accepted for publication Oct 19, 2017.

doi: $10.21037 /$ jtd.2017.11.42

View this article at: http://dx.doi.org/10.21037/jtd.2017.11.42

Lung cancer is one of the most common malignancies and the most common cause of death from cancer worldwide. The estimated number of new cases was 1.8 million for $2012(1)$.

There are no early symptoms, therefore the majority of new cases are diagnosed at an advanced stage of the disease, when treatment with curative intent is a non-applicable strategy. This results in low overall 5-year survival rates (2). Moreover, there are often comorbidities such as COPD and ischemic heart disease that further complicate delivery of care.

On the other hand, early diagnosis is associated with better outcomes. Moreover, delivery of care through multidisciplinary and dedicated, organ-specific tumor boards/teams, and prompt access to all treatment modalities is also linked to improved outcomes. The disease is devastating and lethal and every effort should be made for prevention and early diagnosis as well as access to specialized care. A major concern is cost and smoking bans and screening are indeed cost-effective and life-saving measures. In addition, there must be constant review of old and new treatment modalities and a cost/benefit ratios so that optimal care is delivered but also that health care systems are sustainable long term.

Pulmonologists and medical societies must work to promote strong smoking cessation programs, since cigarette smoke is the most common risk factor for lung cancer. A smoke-free environment would be the best means to prevent most cases of lung cancer. However, even if smoking is totally banned today, there will be cancer cases associated with smoking for at least 20 more years, as the effects on the lung may appear even long after exposure.
Therefore, lung cancer is a mandatory candidate for the development of screening programs, aiming at early stage diagnosis in order to improve cure rates (3).

Numerous studies such as ELCAP, I-ELCAP, DLCST, DANTE, ITALUNG, NELSON trials (4-9) have looked into lung cancer screening and the NLST is the largest study published to date (10). The study showed that $20 \%$ more patients were diagnosed with low dose computed tomography (LDCT), and importantly, 50\% more patients were diagnosed at stage I-II where the disease has high chances of cure (stage I-II: 158 vs. 70 participants in the CT screening vs. the CXR screening groups, and stage IIB to IV: 120 vs. 111 respectively). Although no difference in overall mortality was noted, this is only a 3 -year trial and CXR is also screening (10). So, we can deduce that compared to no screening, LDCT screening saves lives.

Nevertheless, there are important issues to be addressed regarding LDCT screening, including the selection of individuals for screening, false positive results, overdiagnosis and management of positive findings as well as the smooth running of the screening programs coupled with smoking cessation and the support of individuals (11).

False positive findings affect the lives of the screened population and may represent an unnecessary burden for health economics. In the NLST the proportion of false positive findings was $26.6 \%$. A retrospective analysis, using Lung-RADS criteria, reduced these to $12.8 \%$ (12). So, a pre-test calculation of the risk of lung cancer is important.

So far, studies of lung cancer screening have used different criteria for the selection of at risk individuals (examples shown in Table 1). It has also been shown that selecting individuals for screening based on accurate lung 
Table 1 Selection criteria, number of enrolled individuals and the rate of diagnosed lung cancer of major randomized controlled trials [with permission from Kauczor et al. (3)]

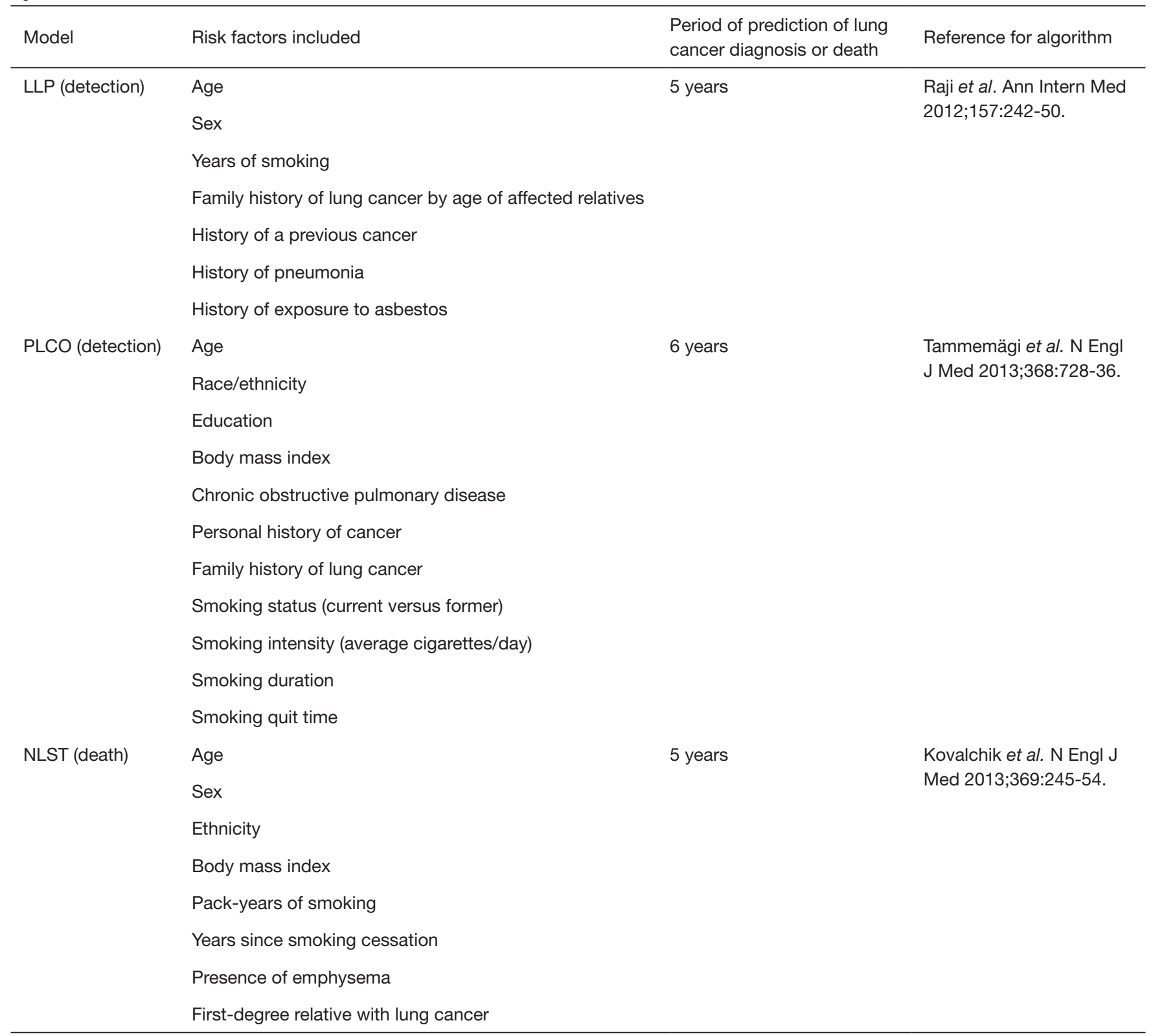

cancer risk prediction models is significantly more sensitive than using the NLST criteria alone $(13,14)$ The Liverpool lung study showed that using a number of risk factors including age, gender, smoking duration, family history of lung cancer before the age of 60 years, a prior diagnosis of cancer or pneumonia may affect the risk of lung cancer up to 27 -fold. So, for example, a 68 -year-old woman with a 25 pack/year history of smoking and no other risk factors, such as prior history of pneumonia, exposure to asbestos or a family history of early age lung cancer, has an absolute risk of lung cancer of $1.5 \%$. On the other hand, a 77-year-old man with a 45 pack/year history of smoking and exposure to asbestos as well as a family history of early age lung cancer has an absolute chance of lung cancer that exceeds $27 \%$ (15).

Overdiagnosis is another unresolved issue in lung cancer screening and it refers to the detection of small lesions confirmed to be malignant but which do not grow, spread, or cause death. This includes patients who are destined to 
die from another cause, e.g., a comorbidity or an unexpected event, in addition to slow growing/non-spreading cancers

Ways to reduce the problems associated with lung cancer screening include: clinical algorithms and clear guidelines, expert centers with high output and expertise, defined radiological protocols, simultaneous use of biomarkers and clear definitions of features of the lesions and their management, stratified by patient risk.

The European Society of Radiology and the European Respiratory Society are recommending lung cancer screening in comprehensive, quality-assured programmes within a clinical trial or in routine clinical practice at certified multidisciplinary medical centres (3). Based on the results and experience of completed and on-going lung cancer screening activities, they suggest the following minimum requirements for the implementation of lung cancer screening:

* Accredited medical centres with multidisciplinary expertise and access to trained professionals, including as a minimum, radiologists, pulmonologists, oncologists, pathologists and chest surgeons;

* Strong smoking cessation programme and experienced staff providing effective cessation and long term abstinence advice;

* Longitudinal comprehensive screening programme throughout the age interval of eligibility, covering the complete protocol, including work-up, follow-up and potential re-entry, also offering an appropriate expectation management. Single-round screening is discouraged;

* Inclusion criteria: age between 55 and 80 years, tobacco smoking history of at least 30 pack-years, and current smoker or ex-smoker who has quit smoking within the last 15 years;

* Exclusion criteria: comorbidities precluding curative therapy and lack of consent to undergo curative therapy;

* Standardised operating procedures for image acquisition, nodule evaluation, positive screening results and their management, monitoring of falsepositive results and rate of iatrogenic complications, and appropriate follow-up;

* Computer-assisted nodule evaluation and documentation. Identical measurement software is required for the follow-up. Volumetric measurements are preferred over diameter measurements;

* Multidetector LDCT with at least 16 detector rows providing isotropic high spatial resolution (slice thickness of about $1 \mathrm{~mm}$ with an increment of 0.7 $\mathrm{mm}$ ) and an effective dose between $1 \mathrm{mSv}$ for normal sized individuals and not more than $3 \mathrm{mSv}$ for obese individuals;

* Collection and submission of lung cancer screening data to a lung cancer screening registry. The set-up of a European lung cancer screening registry including biobank and image bank is recommended;

Moreover, in a recent editorial in the ERf (16), it is suggested that, for successful screening programs, two strategies have to be merged (I) smoking cessation and (II) detection and early treatment of survival-limiting, smokingassociated disease. This should be done by a comprehensive program for high-risk smokers offering a smoking cessation program together with a risk-model based CT-screening of the "Big Three" smoking-associated killer diseases: lung cancer, COPD and cardiovascular disease.

\section{Acknowledgements}

None.

\section{Footnote}

Conflicts of Interest: The authors have no conflicts of interest to declare.

\section{References}

1. Ferlay J, Soerjomataram I, Dikshit R, et al. Cancer incidence and mortality worldwide: sources, methods and major patterns in GLOBOCAN 2012. Int J Cancer 2015;136:E359-86.

2. van Rens MT, de la Rivière $\mathrm{AB}$, Elbers HR, et al. Prognostic assessment of 2,361 patients who underwent pulmonary resection for non-small cell lung cancer, stage I, II, and IIIA. Chest 2000;117:374-9.

3. Kauczor HU, Bonomo L, Gaga M, et al. ESR/ERS white paper on lung cancer screening. Eur Respir J 2015;46:28-39.

4. Stephenson SM, Mech KF, Sardi A. Lung cancer screening with low-dose spiral computed tomography. Am Surg 2005;71:1015-7.

5. Welch HG, Woloshin S, Schwartz LM, et al. Overstating the evidence for lung cancer screening: the International Early Lung Cancer Action Program (I-ELCAP) study. Arch Intern Med 2007;167:2289-95.

6. Wille MM, Dirksen A, Ashraf H, et al. Results of the 
Randomized Danish Lung Cancer Screening Trial with

Focus on High-Risk Profiling. Am J Respir Crit Care Med 2016;193:542-51.

7. Infante $\mathrm{M}$, Cavuto $\mathrm{S}$, Lutman FR, et al. A randomized study of lung cancer screening with spiral computed tomography: three-year results from the DANTE trial. Am J Respir Crit Care Med 2009;180:445-53.

8. Lopes Pegna A, Picozzi G, Mascalchi M, et al. Design, recruitment and baseline results of the ITALUNG trial for lung cancer screening with low-dose CT. Lung Cancer 2009;64:34-40.

9. Horeweg N, van Rosmalen J, Heuvelmans MA, et al. Lung cancer probability in patients with CT-detected pulmonary nodules: a prespecified analysis of data from the NELSON trial of low-dose CT screening. Lancet Oncol 2014;15:1332-41.

10. National Lung Screening Trial Research Team, Church TR, Black WC, et al. Results of initial low-dose computed tomographic screening for lung cancer. $\mathrm{N}$ Engl J Med 2013;368:1980-91.

Cite this article as: Bostantzoglou C, Kontogianni C, Iliopoulou M, Gaga M. Best of lung cancer session presented during ERS 2017 Chinese language day: lung cancer screening. J Thorac Dis 2017;9(Suppl 16):S1563-S1566. doi: 10.21037/ jtd.2017.11.42
11. National Lung Screening Trial Research Team, Aberle DR, Adams AM, et al. Reduced lung-cancer mortality with low-dose computed tomographic screening. $\mathrm{N}$ Engl J Med 2011;365:395-409.

12. Pinsky PF, Gierada DS, Black W, et al. Performance of Lung-RADS in the National Lung Screening Trial: a retrospective assessment. Ann Intern Med 2015;162:485-91.

13. Tammemägi MC, Katki HA, Hocking WG, et al. Selection criteria for lung-cancer screening. N Engl J Med 2013;368:728-36.

14. Kovalchik SA, Tammemagi M, Berg CD, et al. Targeting of low-dose CT screening according to the risk of lungcancer death. N Engl J Med 2013;369:245-54.

15. Cassidy A, Myles JP, van Tongeren M, et al. The LLP risk model: an individual risk prediction model for lung cancer. Br J Cancer 2008;98:270-6.

16. Kauczor HU, Heussel CP, von Stackelberg O. Time to take CT screening to the next level? Eur Respir J 2017;49. pii: 1700064. 